\title{
New Dwarf Galaxies in the IC342/Maffei Group
}

\author{
W. K. Huchtmeier \\ Max-Planck-Institut für Radioastronomie, Bonn, D-53121, Germany \\ I. D. Karachentsev \\ Special Astrophysical Observatory (SAO), N. Arkhys, Russia \\ V. E. Karachentseva \\ Astronomical Observatory of Kiev State University, Kiev, Ukraine
}

\begin{abstract}
This is a report on the detection of $\mathrm{HI}$ emission from three 'new' dwarf galaxies Perseus A, Perseus B, and Camelopardalis D in the IC342/Maffei group of galaxies and of Draco A. The actual number of (probable) member galaxies of this group increases to 19 galaxies. Its velocity dispersion is $86 \mathrm{~km} \mathrm{~s}^{-1}$. With a distance of $2.2 \pm 0.5 \mathrm{Mpc}$ this group is the nearest to the Local Group and might have considerable dynamical influence on the Local Group.
\end{abstract}

\section{Introduction}

Due to its position within the zone of avoidance between the Andromeda region of the Local Group and the M81 group, IC342/Maffei has been recognized only recently as a group. Since 1994 a great number of dwarf galaxies have been discovered in this area because of growing interest for galaxies in the zone of avoidance. There have been blind $\mathrm{H}$ I surveys and optical searches for galaxies in the area. The recent discoveries of Dwingeloo 1 (Kraan-Korteweg et al. 1994, Huchtmeier et al. 1995), Dwingeloo 2 (Burton et al. 1996), Cas 1 (Huchtmeier et al. 1995), MB1 and 2 (McCall and Buta 1995, McCall et al. 1995), Cam B (Huchtmeier et al. 1997), MB3 (McCall and Buta 1997) have increased the number of known galaxies in this group considerably. Here we report $\mathrm{H}$ I-detection of the dwarf galaxies Camelopardalis D, Perseus A and B, and of Draco A in the area of the M81 group. ${ }^{1}$ The IC342/Maffei group is the nearest group outside the Local Group with a photometric distance of $2.2 \mathrm{Mpc}$.

A new list of candidates of nearby dwarf galaxies from the sky survey of surface brightness dwarf galaxies based on the POSS II and ESO/SERC films has been searched for $\mathrm{HI}$ emission with the 100-m radio telescope at Effelsberg. So far two lists of the Karachentsev survey have been published (Karachentseva and Karachentsev 1998, Karachentseva et al. 1999), H I observations of some of these

\footnotetext{
${ }^{1}$ More possible detections have been reported by Rivers (1998)
} 

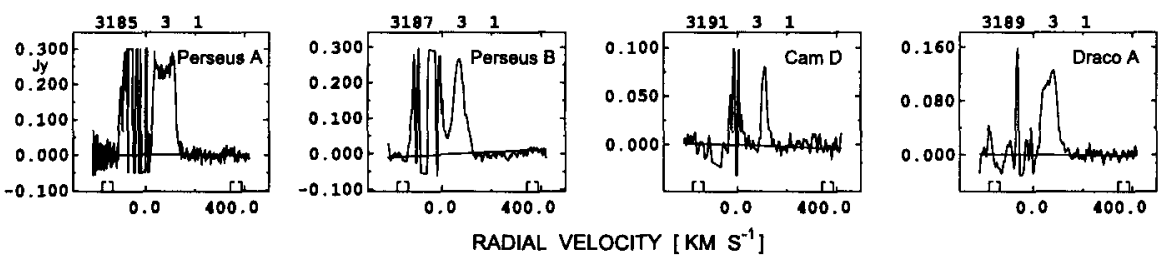

Figure 1. H I profiles of the 4 'new' dwarf galaxies in the area of the IC342/Maffei group observed with the $100-\mathrm{m}$ radio telescope at Effelsberg, from left to right : Perseus A, Perseus B, Camelopardalis D, and Draco 1. All profiles but Perseus A have been Hanning smoothed. The channel spacing is $2.6 \mathrm{~km} \mathrm{~s}^{-1}$.

new dwarf galaxies have been reported (Huchtmeier et al. 1997, Huchtmeier et al. 1999). Among those newly discovered galaxies three are situated in the IC342/Maffei group according to their position and radial velocity.

\section{Observations}

Observations were performed with the 100-m radio telescope at Effelsberg which has a half power beam width (HPBW) of 9.3' at the wavelength of 21-cm. Observations have been obtained in the total power mode combining the onsource position with a reference field. A bandwidth of $3.125 \mathrm{MHz}$ was split into four channels yielding a channel spacing of $12.2 \mathrm{kHz}$ and a resolution of 3.1 $\mathrm{km} \mathrm{s}^{-1}$ (or $5.1 \mathrm{~km} \mathrm{~s}^{-1}$ after Hanning smoothing). For all galaxies four additional positions one beam width off the central position in R.A. and Dec. have been observed to check for extent of the $\mathrm{HI}$. The $\mathrm{HI}$ emission was centered on the optical positions and was hardly more extended than the HPBW. The profiles are shown in Fig. 1, the observed H I parameters in Table 1. Apart from Cam D all profiles seem partially confused by local $\mathrm{HI}$ which is seen best for Perseus $\mathrm{A}$ and $B$.

Table 1. H I parameters of the 'new' dwarf galaxies in the area of the IC342/Maffei Group

\begin{tabular}{|c|c|c|c|c|c|c|}
\hline Name & R.A. (1950.0 & Dec. & velocity & H I-flux & $\mathrm{S}_{\max }$ & line-width \\
\hline & & & $\mathrm{kms} \mathrm{s}^{-1}$ & $\mathrm{Jy} \mathrm{km} \mathrm{s}^{-1}$ & Jy & $50 \% 20 \%$ \\
\hline Perseus A & $\begin{array}{llll}02 & 21 & 03.7\end{array}$ & +553709 & $75 \pm 1$ & 24.2 & $0.29 \pm 0.025$ & 94104 \\
\hline Perseus B & 022351.3 & +571550 & $72 \pm 2$ & 14.0 & $0.26 \pm 0.016$ & 4686 \\
\hline Cam D & 055323.1 & +732524 & $111 \pm 2$ & 2.1 & $0.08 \pm 0.008$ & 2334 \\
\hline Draco A & $\begin{array}{lll}12 & 1142.2\end{array}$ & +662212 & $66 \pm 1$ & 8.1 & $0.13 \pm 0.012$ & 6881 \\
\hline
\end{tabular}


Table 2. Galaxies in the IC342/Maffei Group

\begin{tabular}{|c|c|c|c|c|c|c|}
\hline Name & Type & $\begin{array}{c}\text { optical } \\
\text { diameter }\end{array}$ & $\begin{array}{c}\text { velocity } \\
\mathrm{v}_{0}\end{array}$ & $M_{B}$ & $M_{H I}$ & $M_{H I} / L_{B}$ \\
\hline & & arc min & $\mathrm{km} \mathrm{s}^{-1}$ & & $10^{7} M_{\odot}$ & \\
\hline 1 & 2 & 3 & 4 & 5 & 6 & 7 \\
\hline IC342 & Scd & 21.420 .9 & 229 & -20.3 & 355 & 0.34 \\
\hline Maffei I & $\mathbf{E}$ & $\begin{array}{ll}3.3 & 1.7\end{array}$ & 224 & -19.5 & & \\
\hline Maffei II & Sbc & $\begin{array}{ll}5.8 & 1.6\end{array}$ & 209 & -19.5 & 35 & 0.04 \\
\hline Dw1 & SBcd & 4.20 .3 & 309 & -17.5 & 24 & 0.95 \\
\hline NGC 1569 & IBm & $\begin{array}{ll}3.6 & 1.8\end{array}$ & 87 & -17.1 & 8 & 0.05 \\
\hline NGC 1560 & Sd & 11.60 .8 & 164 & -15.5 & 27 & 1.28 \\
\hline UGCA 105 & $\operatorname{Im} ?$ & 5.53 .5 & 264 & -15.3 & 20 & 1.12 \\
\hline UGCA 92 & Im? & 2.01 .0 & 66 & -14.0 & 6 & 0.49 \\
\hline UGCA 86 & $\operatorname{Im} ?$ & $\begin{array}{ll}0.8 & 0.7\end{array}$ & 262 & -12.7 & 8 & 0.93 \\
\hline Cas 1 & dIm & 1.91 .6 & 264 & -14.8 & 5 & 0.39 \\
\hline MB1 & Sd & 6.01 .0 & 398 & -12.5 & 2.2 & 1.44 \\
\hline Dw2 & Im & 6.4 & 291 & -13.1 & 4.1 & 1.49 \\
\hline Cam B & Im & 2.21 .1 & 264 & -12.1 & 0.3 & 0.28 \\
\hline Cam D & $\operatorname{Im}$ & $\begin{array}{lll}0.6 & 0.5\end{array}$ & 278 & -11.0 & 0.12 & 0.29 \\
\hline Perseus A & Im & 1.40 .8 & 288 & -12.9 & 1.4 & 0.58 \\
\hline Perseus B & Im & 1.70 .5 & 283 & -12.7 & 1.2 & 0.66 \\
\hline Cam C & $\mathrm{dIm}$ & 1.80 .4 & 151 & & & \\
\hline Cam A & dSph & 3.72 .1 & & & & \\
\hline MB3 & $\mathrm{dSph}$ & 1.60 .5 & & & & \\
\hline
\end{tabular}

\section{Discussion}

The clustering of galaxies around IC342 and Maffei 1 (Fig. 2) within the zone of avoidance along the supergalactic equator in addition to the similar corrected radial velocities (Table 1) suggests strongly a typical group of galaxies. In the past greatly different distances have been quoted for this group, see discussion by Krismer et al. (1995) and by McCall and Buta (1997). In recent years photometric distances have been derived for 10 galaxies in this group (Karachentsev and Tikhonov 1993, 1994, Karachentsev et al. 1997, and unpublished work). These distances agree quite well with each other and yield a distance of $2.2 \pm 0.5$ $\mathrm{Mpc}$ for the IC342/Maffei group. At such a close distance it might have played a significant role in the dynamical evolution of the Local Group (McCall 1986, 1989, Zheng et al. 1991, Valtonen et al. 1993, Peebles 1994).

Acknowledgments. The NED database is supported at IPAC by NASA.

This work has been partially supported by the Deutsche Forschungsgemeinschaft (DFG) under project no. 436 RUS 113/470/0.

\section{References}

Burton, W. B., Verheijen, M. A. W., Kraan-Korteweg, R. C., Henning, P. A. 1996, A\&A, 309, 687 


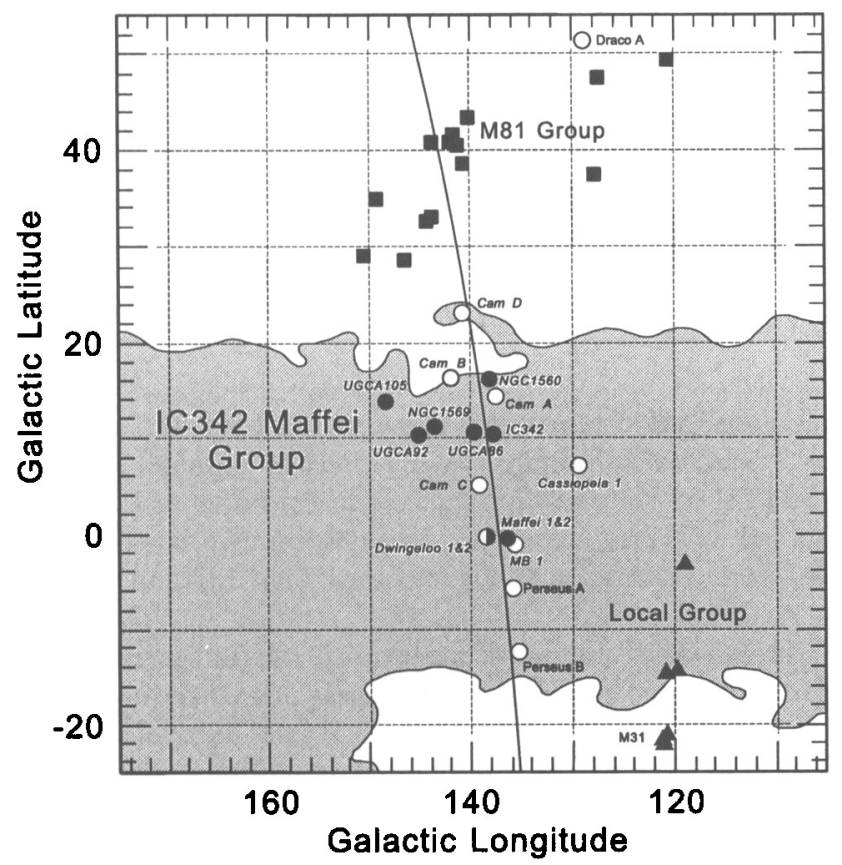

Figure 2. The distribution of galaxies in the area of the IC342/Maffei group (indicated by circles) along the supergalactic equator in galactic coordinates. Galaxies detected since 1994 are shown by open circles.

Huchtmeier, W. K., Lercher, G., Seeberger, R., Saurer, W., Weinberger, R. 1995, A\&A, 293, L33

Huchtmeier, W. K., Karachentsev, I. D., Karachentseva, V. E. 1997, A\&A, 322, 375

Karachentseva, V. E., Karachentsev, I.D. 1998, A\&A Suppl., 127, 409

Karachentseva, V. E., Karachentsev, I. D., Richter, G. M. 1999, A\&A Suppl., 135,221

Karachentsev, I. D., Drozdovsky, I., Kajsin, S., Takalo, L. O., Heinämäki, P., Valtonen, M. 1997, A\&A Suppl. 124, 559

Karachentsev, I. D., Makarov, D. A. 1996, AJ, 111, 794

Karachentsev, I. D., Tikhonov, N. A. 1993, A\&A Suppl. 100, 227

Karachentsev, I. D., Tikhonov, N. A. 1994, A\&A, 286, 718

Kraan-Korteweg, R. C., Loan, A. J., Burton, W. B., Lahav, O., Ferguson, H. C., Henning, P. A., Lynden-Bell, D. 1994, Nature, 372, 77

Krismer, M., Tully, R. B., Gioia, I. M. 1995, AJ, 110, 1584

Lynden-Bell, D., \& Wood, R. 1968, MNRAS, 138, 495 
McCall, M. L., 1986, JRASC, 80, 271

McCall, M. L., 1989, AJ, 97, 1341

McCall, M. L., Buta, R. J. 1995, AJ, 109, 2460

McCall, M. L., Buta, R. J., Huchtmeier, W. K. 1995, IAU Circ. No. 6159

Peebles, P. J. E. 1994, ApJ, 429, 43

Rivers, A. J., 1998, AAS, 193, 28.05

Valtonen, M. J., Byrd, G.G., McCall, M. L., Innanen, K. A. 1993, AJ, 105, 886

Zheng, J. Q., Valtonen, M. J., Byrd, G. G., 1991 A\&A 247, 20 